\title{
Is It Statin Induced Polymyalgia Rheumatica or Just a Coincidence?
}

\author{
Şule ŞAHINN ONAT, Seda BİÇER, Zehra ŞAHİN, Sibel ÖZBUDAK DEMİR \\ Department of Physical Medicine and Rehabilitation, \\ Ankara Physical Medicine and Rehabilitation Training and Research Hospital, Ankara, Turkey
}

A 53-year-old female presented with pain in her bilateral shoulders, hips and neck, stiffness (lasting one hour), and proximal weakness for three months. She declared that her symptoms had ensued one week after the usage of daily $40 \mathrm{mg}$ atorvastatin for hyperlipidemia. As such, atorvastatin was discontinued; however, her complaints did not decline. Physical examination revealed painful and restricted shoulder and neck movements, weakness in shoulder and hip muscles, and a swollen, warm right knee. Laboratory investigations including creatine kinase $(\mathrm{CK})$, rheumatoid factor and anti-nuclear antibody levels were within normal values, except for the increased C-reactive protein $(0.744 \mathrm{mg} / \mathrm{dL}, \mathrm{N}: 0-0.5)$ and erythrocyte sedimentation rate $(75 \mathrm{~mm} / \mathrm{h}$, $\mathrm{N}$ : 0-20). X-rays of the hand, knee, hip and shoulder were non-contributory. Ultrasound illustrated biceps tenosynovitis and trochanteric bursitis bilaterally, and increased suprapatellar fluid in right knee (Figure 1). Overall, the patient was diagnosed with polymyalgia rheumatica (PMR) according to the 2012 European League Against Rheumatism/American College of Rheumatology classification criteria. $^{1} \mathrm{Her}$ symptoms and clinical findings (erythrocyte sedimentation rate and C-reactive protein levels) responded to medium dose of prednisolone $(20 \mathrm{mg} / \mathrm{d})$ treatment dramatically.
Although statins have been reported to be associated with some musculoskeletal side effects such as myalgia, myositis, myopathy, and elevated $\mathrm{CK}$, statin induced PMR is a very rare condition which has been reported only in a few anecdotal reports. ${ }^{2-4}$ Likewise, statin treatment might trigger the development of PMR in our patient. Since statin induced PMR and myalgia have similar clinical features, the exact diagnosis can be challenging. Statin induced myalgia presents with/without increased $\mathrm{CK}$ levels with normal erythrocyte sedimentation rate and $\mathrm{C}$-reactive protein levels. Therefore, patients with statin induced myalgia may have normal serum CK levels, which sometimes make it difficult to distinguish PMR from myopathy syndromes. In case of drug discontinuation, symptoms heal in a period of one-week to four-month in statin, myopathy and treatment of steroid is rarely required. ${ }^{5}$ PMR patients do not recover spontaneously and corticosteroid treatment is usually needed. ${ }^{6}$

Additionally, patients with PMR were more likely to have abnormal ultrasound findings in their shoulders (especially subdeltoid bursitis and biceps tenosynovitis), and hips (synovitis and/or trochanteric bursitis). ${ }^{1}$ Therefore, these findings can be useful in discriminating PMR from other shoulder/hip pathologies and in evaluating the patient for classification criteria of PMR. 

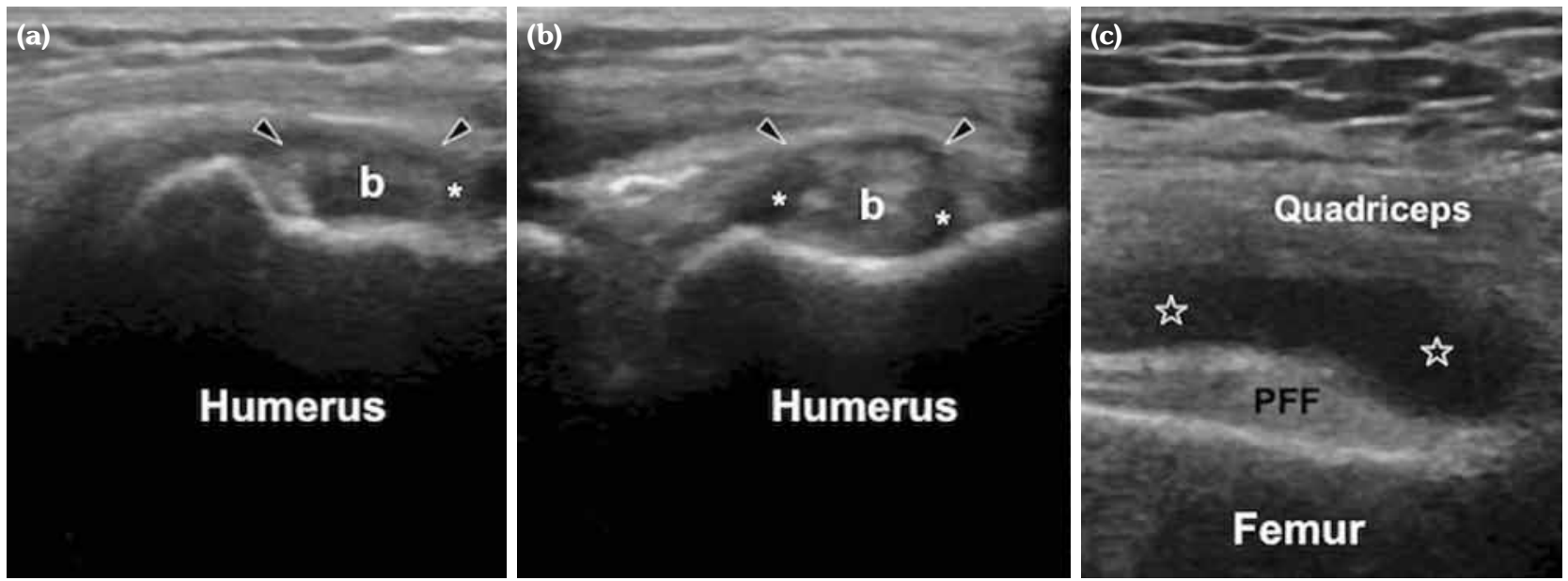

Figure 1. (a, b) Ultrasound imaging (axial views) over the long head of the biceps tendons shows hypoechoic fluid surrounding the tendons (white asterisks), edema and heterogeneous echotexture (arrowheads) on both sides. (c) Ultrasound imaging (longitudinal view) shows increased suprapatellar fluid in the right knee (void stars). b: Biceps tendon; PFF: Prefemoral fat pad.

On the other hand, it has been discussed whether there is an association between the use of statin treatment and the occurrence of PMR in the spontaneous reporting database of the World Health Organization. ${ }^{7}$ In that study, authors declared that the use of a statin might be associated with the occurrence of PMR. It has also been reported that statins may induce neoantigens as a result of muscle damage that are subsequently presented to the immune system. ${ }^{8}$ A similar mechanism may be responsible in statin induced PMR.

We highlight the relevant association between the statin treatment and the development of PMR. Their coexistence may cause diagnostic and/or therapeutic challenges for prompt management. Finally, we would like to underscore the role of ultrasound -an inexpensive and convenient method- that also provides additional value for PMR evaluation.

\section{Declaration of conflicting interests}

The authors declared no conflicts of interest with respect to the authorship and/or publication of this article.

\section{Funding}

The authors received no financial support for the research and/or authorship of this article.

\section{REFERENCES}

1. Dasgupta B, Cimmino MA, Maradit-Kremers H, Schmidt WA, Schirmer M, Salvarani C, et al. 2012 provisional classification criteria for polymyalgia rheumatica: a European League Against Rheumatism/ American College of Rheumatology collaborative initiative. Ann Rheum Dis 2012;71:484-92.

2. Goëb V, Guillemant N, Vittecoq O, Le Loët X. Cerivastatin-induced polymyalgia rheumatica-like illness. Clin Rheumatol 2004;23:179.

3. Kay J, Finn DS, Stone JR. Case records of the Massachusetts General Hospital. Case 4-2006. A 79-year-old woman with myalgias, fatigue, and shortness of breath. N Engl J Med 2006;354:623-30.

4. Rudski L, Rabinovitch MA, Danoff D. Systemic immune reactions to HMG-CoA reductase inhibitors. Report of 4 cases and review of the literature. Medicine (Baltimore) 1998;77:378-83.

5. Harper CR, Jacobson TA. The broad spectrum of statin myopathy: from myalgia to rhabdomyolysis. Curr Opin Lipidol 2007;18:401-8.

6. Kermani TA, Warrington KJ. Advances and challenges in the diagnosis and treatment of polymyalgia rheumatica. Ther Adv Musculoskelet Dis 2014;6:8-19.

7. de Jong HJ, Saldi SR, Klungel OH, Vandebriel RJ, Souverein PC, Meyboom RH, et al. Statin-associated polymyalgia rheumatica. An analysis using WHO global individual case safety database: a case/noncase approach. PLoS One 2012;7:e41289.

8. Grable-Esposito P, Katzberg HD, Greenberg SA, Srinivasan J, Katz J, Amato AA. Immune-mediated necrotizing myopathy associated with statins. Muscle Nerve 2010;41:185-90. 